\title{
Challenges for Detection of Elderly Abuse in Teaching Hospitals: A Interventional Study to Improve the Knowledge of Health Care Providers
}

\author{
Akram Fallah Shojaei Mardomakdehi ${ }^{1}$, Shima Sum ${ }^{2}$ and Mahbobeh Faramarzi ${ }^{2,{ }^{*}}$ \\ ${ }^{1}$ Student Research Committee, Health Research Institute, Babol University of Medical Sciences, Babol, Iran \\ ${ }^{2}$ Social Determinants of Health Research Center, Health Research Institute, Babol University of Medical Sciences, Babol, Iran \\ "Corresponding author: Social Determinants of Health Research Center, Health Research Institute, Babol University of Medical Sciences, Babol, Iran. Email: \\ mahbob330@yahoo.com
}

Received 2019 March 07; Revised 2019 June 13; Accepted 2019 June 30.

\begin{abstract}
Objectives: Elder abuse is a serious and dangerous form of domestic violence that is of particular concern to communities and healthcare professionals. Since health care providers have a central position for detecting elder abuse in hospitals, this study was conducted to determine the barriers in identifying elder abuse among health care providers and the effect of intervention to deal with the problem.

Methods: The participants completed the health care providers' awareness on reporting the elder abuse questionnaire. Then, the interventional study was conducted on 235 health care providers at two teaching hospitals of Babol University of Medical Sciences. The intervention was conducted as a one-day workshop by two professors for four hours and included theoretical and practical subjects. Two months after implementing the protocol for detecting and screening elder abuse in hospital wards, the health care providers completed three questionnaires, including the awareness questionnaire, the potential barriers to assessing elder abuse in hospitals, and the health care providers' implementation problems in detecting and reporting elder abuse.

Results: The mean health care providers' awareness to detect elder abuse improved significantly after intervention $(8.51 \pm 2.81 \mathrm{vs.}$ $15.53 \pm 2.39 ; \mathrm{P}<0.001)$. The challenges of abuse detection were; lack of awareness about elder abuse and its prevalence $(54.8 \%)$, fear of legal consequences (51.9\%), and fear of communication problems between older adults and their family (53.6\%).

Conclusions: More frequent and pragmatic education is necessary to strengthen the practical knowledge of health care providers in regards to the detection of elder abuse.
\end{abstract}

Keywords: Elder Abuse, Identification, Challenges, Intervention

\section{Background}

The most important problem for identifying elder abuse is the lack of clear definition of the term "abuse" and its types (1). There are no standard definitions for the concept of "elder abuse" agreed upon by all, as existing definitions are often based on specific perspectives and the professional needs of different scholars (2). According to the WHO, elder abuse is a single act, series of acts, or lack of appropriate action in any relationship where there is an expectation of trust that cause harm, pain, distress, anxiety, and discomfort to the older adult. Elder abuse can take various forms, such as physical, psychological, sexual, financial abuse, and neglect (3). Elder abuse is not a new issue, however, it has recently been raised as a major public health problem and a widespread and growing social prob- lem around the world that is intentionally or unintentionally inflicted on older adults and causes harm (4).

Although some studies reported the prevalence of abuse in the Iranian population $(5,6)$ the report of elder abuse is varied in different studies (7). WHO estimates the rate of domestic elder abuse as $1 \%$ to $35 \%$ (8). Previous studies reported that $73.3 \%$ of all abusive behaviors toward older adults occur at home (9). Although there are some researches about medical illness in Iranian elderly $(10,11)$, there are no precise statistics about the prevalence of elder abuse (12). Few studies on the subject have been conducted from a medical point of view with an emphasis on eliminating or preventing physical pain (13).

One of the most difficult clinical challenges in gerontology is the detection of elder abuse, in which health care providers play a key role. Health care providers com- 
municate with all the sectors of the society, and therefore, play a vital role in breaking the chain of violence in the life of older adults and preventing its many complications in the community. Noting the role of health care providers in this area is essential (14). Through active participation in different areas of life and family environment, health care providers might be better able than other members of healthcare teams to detect such problems and treat misconduct that leads to decreased self-esteem, frustration, incompetence, and disability in older adults (15). Winterstein emphasized that nursing education about neglecting older adults should be further challenged and training programs should be arranged to raise awareness about this issue (16). Phelan stated that health care providers play a distinct role in providing secondary care to older adults and paying home visits to older adults is very important for detecting misconduct. In tertiary care, health care providers can monitor situation of older adult's at home and create conditions that prevent elder abuse (17). Educational programs for those working with older adults increase their skills in detecting misconduct and taking appropriate measures to handle both the abused and the abusers. Increasing employee awareness about elder abuse and taking legal actions help resolve ethical problems such as elder abuse (18). Fisher et al. showed that detecting elder abuse is vital and that health professionals should seek to detect and report any such cases. Fisher's study revealed several barriers to the detection of elder abuse by medical students. Medical professors who are responsible for educating the students on elder abuse should ascertain that their students have fully understood their responsibilities in this area as physicians. Finally, Fisher et al. concluded that simulation can provide a useful educational tool in this area (19).

Since Iranian health providers lack adequate awareness about the issue of elder abuse, to prevent elder abuse and promote the health of older adults, families and the entire society develop global health policies and plans.

\section{Objectives}

The present interventional study was conducted with two main objectives. The first aim was to evaluate the effect of an interventional plan to health care provider's empowerment on identification of elder abuse. The second aim was to identify the challenges for detection of elderly abuse in teaching hospitals.

\section{Methods}

The method of the study comprised of two sections: interventional and descriptive. The interventional section of the study was conducted on all health care providers at Ayatollah Rouhani and Shahid Beheshti teaching hospitals in Babol, Iran, (except for gynecology and pediatric health care providers who did not deal with elderly care) using census sampling. The participants were invited to attend an elder abuse detection empowerment workshop. The number of eligible health care providers in the two hospitals was $395 ; 235$ of those who were on the modular inservice training list were ultimately included in the study. All 235 final candidates were accepted to participate in the study. Before beginning the intervention, the participants were briefed on the research objectives and gave their informed consent. The health care providers' empowerment intervention was conducted in six groups of about 45 persons. In the first stage, the health care providers attended a four-hour training workshop; before the workshop, they were given a questionnaire that assessed their awareness and views on the detection and reporting of elder abuse. The participants were briefed on the study objectives and ensured of the confidentiality of their information and ideas before beginning data collection. The participants then gave their consent and proceeded to filling out the questionnaires in a maximum of 20 minutes. They then received theoretical and practical training. The theoretical training covered concepts such as aging, normal changes associated with aging, elderly rights, elder abuse and the need to emphasize the phenomenon, types of elder abuse, preventive measures, timely detection, and means of reporting abuse. In addition to these theoretical issues, a practical workgroup was held to present scenarios of health care providers on how to use diagnostic tools and report elder abuse as well as refer suspected cases to the authorities. The health care providers were oriented with pamphlets on the subject. A protocol was ultimately presented for the timely detection and reporting of elder abuse in hospitals. The screening protocol instructed on referring possible cases of elder abuse to a higher authority and what interventions to take in the case of sufficient evidence of abuse.

In the descriptive section of the study, the health care providers completed the health care providers' awareness on reporting elder abuse questionnaire before the intervention. Two months after implementation of elder abuse detection and screening protocol in the hospital wards, the health care providers completed the awareness questionnaire (the awareness and knowledge tool) once again. To identify the barriers of elderly abuse, health care providers completed the potential barriers for assessing elder abuse in hospitals and the health care providers' implementation problems in detecting and reporting elder abuse two months after the intervention.

The project's compliance with the ethical code was ap- 
proved by the Ethics Committee of Babol University of Medical Sciences (MUbabol.HRI.REC.1394.15).

\subsection{Instruments}

3.1.1. The Researcher-Made Health Care Providers' Awareness on Reporting Elder Abuse Questionnaire

This tool has 21 items that address health care providers' attitudes and practices with regard to detecting and managing elder abuse, including the tool assessing health care providers' awareness on a threepoint Likert-type scale (true, not sure, and false). The validity of the tool was approved by geriatricians and its reliability was reported as 0.81 in the study.

\subsubsection{The Potential Barriers to Assessing Elder Abuse in Hospi-} tals Questionnaire

This tool has 14 items that evaluate the health care providers' perspective on the potential barriers to their assessment of elder abuse, including "recent changes to the rules and guidelines", "the lack of professional protocols for evaluating elder abuse", and "the lack of professional protocols for managing abuse". This tool identified the importance of each barrier in evaluating abuse. The validity of the tool was approved by gerontologists and its reliability was reported as 0.92 in the study.

\subsubsection{The Health Care Providers' Implementation Problems in Detection and Reporting Elder Abuse Questionnaire}

This researcher-made tool has eight items, which assess the health care providers' performance in terms of detecting and reporting elder abuse on a three-point scale (poor, good, and excellent). The validity of the tool was approved by the gerontologists.

\section{Results}

The health care providers who entered the study were $86 \%$ females and $14 \%$ males with of mean age of $35.15 \pm$ 7.68 (range: 21 - 55). A total of $79 \%$ of the participants were married and $94.4 \%$ had a bachelor degree. The health care providers' mean work experience was $7.7 \pm 6.61$ years. Their minimum work experience was one year (12.1\%) and the highest was 29 years (0.8\%). A majority of the health care providers were employed in the surgery ward (19.7\%) and a very small minority were employed in the endoscopy ward (0.85\%). Most of the study subjects (91.49\%) had never attended classes or workshops on elder abuse. Most health care providers (91.49\%) claimed that there were no protocols for elder abuse in the hospital.

Generally, the findings of this study showed that the mean score of health care providers regarding elder abuse

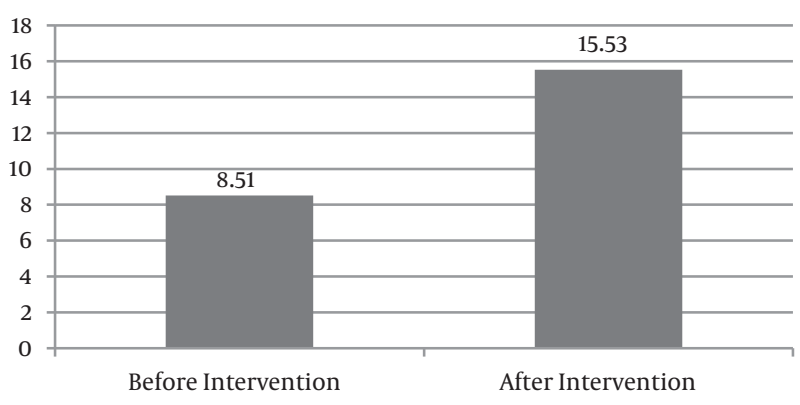

Figure 1. Healthcare providers' awareness score before and after the elder abuse intervention

was low (Figure 1). Only one-third (17.9\%) of the health care providers stated that older adults were severely abused or neglected. Less than half (41.7\%) thought that health care providers could detect abuse. Only one-fifth (20.4\%) knew that most cases of abuse occur in elderly care centers. Few health care providers (13.6\%) knew that they would be supported by the government in case of threats following the reporting of elder abuse, and less than half (43.4\%) were aware that older adults are at a higher risk of abuse than children (Table 1).

The health care providers' awareness was also examined by four scenarios, which will be discussed here in detail. The first scenario dealt with physical abuse and its findings showed that 216 of the health care providers (91.9\%) were able to detect abuse and 87 (39.5\%) were able to detect the type of abuse. Most of the health care providers $s(n=166,71.6 \%)$ were aware of the important effects of abuse. Regarding what construed abuse, all health care providers considered the throwing of an object toward a mother and disrespecting as an instance of abuse. A total of $151(65.1 \%)$ said that they helped someone who needed help in these situations. The second scenario dealt with financial abuse and its findings showed that 215 (91.9\%) of the health care providers were able to detect abuse and 155 (70.5\%) were able to detect the type of abuse. The third scenario dealt with cognitive abuse and its findings showed that 223 (94.9\%) health care providers were able to detect abuse. The fourth scenario also dealt with physical abuse and its findings showed that $82(34.9 \%)$ of the health care providers were able to detect abuse and 15 (6.3\%) were able to detect the type of abuse.

The results showed that the educational intervention influenced the health care providers' awareness ( $\mathrm{P}=$ 0.001). There was a weak association between the health care providers' awareness and age $(\mathrm{r}=-0.130$ and $\mathrm{P}=$ 0.04 ), as younger health care providers had higher levels of awareness. The health care providers at Rouhani Hos- 


\begin{tabular}{|c|c|c|c|}
\hline Items & True & Not Sure & False \\
\hline Older adults are rarely subjected to abuse or neglect & $42(9.17)$ & $122(9.51)$ & $71(2.30)$ \\
\hline There are clear definitions of elder abuse & $69(4.29)$ & $106(1.45)$ & $60(5.25)$ \\
\hline Nurses can correctly detect elder abuse & $98(7.41)$ & $114(5.48)$ & $23(8.9)$ \\
\hline Iran's health system has a specific reporting protocol for elder abuse, just like the child abuse reporting protocol & $43(3.18)$ & $111(2.47)$ & $81(5.34)$ \\
\hline Most cases of elder abuse occur in elder care centers & $48(4.20)$ & $124(8.52)$ & $63(8.26)$ \\
\hline Most elder abuse cases are never detected or reported by nurses & $55(4.23)$ & $144(3.61)$ & $36(3.15)$ \\
\hline Most cases of elder abuse include only minor damage & $30(8.12)$ & $123(3.52)$ & $82(9.34)$ \\
\hline There are enough resources in Iran to support older adults in cases of elder abuse & $24(2.10)$ & $115(9.48)$ & $96(9.40)$ \\
\hline Most older adults can ask for help if subjected to abuse & $48(4.20)$ & $100(6.42)$ & $87(0.37)$ \\
\hline I will be supported by the government in the case of threats following the reporting of elder abuse & $32(6.13)$ & $130(3.55)$ & $73(1.31)$ \\
\hline The victim of abuse should consent to reporting the abuse & $87(0.37)$ & $106(1.45)$ & $42(9.17)$ \\
\hline The mandatory reporting of elder abuse is not my responsibility & $58(7.24)$ & $99(1.42)$ & $78(2.33)$ \\
\hline A major reason for not reporting abuse is the possibility of long legal proceedings & $96(9.40)$ & $108(0.46)$ & $31(2.13)$ \\
\hline Immediate action will be taken if a case of abuse is reported & $96(9.40)$ & $108(0.46)$ & $31(2.13)$ \\
\hline Reporting abuse is a violation of the victims' rights & $43(3.18)$ & $97(3.41)$ & $95(4.40)$ \\
\hline The mandatory reporting of suspected cases of abuse is an effective way for dealing with this problem & $98(7.41)$ & $110(8.46)$ & $27(5.11)$ \\
\hline Older adults are more likely to be abused than children & $102(4.43)$ & $104(3.44)$ & $29(3.12)$ \\
\hline Reporting a case of abuse makes the abuser more aggressive & $96(9.40)$ & $98(7.41)$ & $41(4.17)$ \\
\hline Reporting abuse can damage the nurse-patient relationship & $34(5.14)$ & $120(1.51)$ & $81(5.34)$ \\
\hline Victims of abuse often deny that they have been abused & $80(0.34)$ & $117(8.49)$ & $38(2.16)$ \\
\hline Reporting abuse is a violation of elderly rights & $40(0.17)$ & $88(4.37)$ & $107(5.45)$ \\
\hline
\end{tabular}

${ }^{\mathrm{a}}$ Values are expressed as No. (\%).

pital had a more favorable attitude toward older adults ( $\mathrm{r}$ $=0.160$ and $\mathrm{P}=0.0015)$. Health care providers with more work experience had a lower level of awareness $(\mathrm{P}=0.004$ and $\mathrm{r}=-0.187)$. The multivariate regression analysis showed that the only predictive factor of health care providers' awareness before training was the place (i.e. hospital) of work $(\beta=0.218$ and $\mathrm{P}=0.005)$, as the awareness of health care providers working at Rouhani Hospital was higher than those observed in the health care providers working at the Shahid Beheshti Hospital.

Examining the causes and barriers of abuse revealed the lack of awareness about abuse and its prevalence (54.8\%), fear of legal consequences (summons to court, bad reputation, and scandal) (51.9\%), and fear of communication problems between older adults and their family (53.6\%) as the most important barriers for assessing elder abuse in hospital wards (Table 2 and Figure 2).

The findings on the implementation problems faced by health care providers in the two months of their elder abuse screening, detecting, and reporting in hospital wards showed that less than one-third of them believe that wards offer a proper space for assessing older adults and more than half $(63.3 \%)$ believe that it is never possible to access older adults alone in the ward without the presence of their relatives (Figure 3).

\section{Discussion}

The aim of this study was to determine the barriers of identification of elder abuse among health care providers and improve the situation through educational interventions. According to the results, health care providers had a poor awareness about elder abuse that reached a desirable level after training. The findings are consistent with the results of many studies. A study showed that health care providers and physicians do not have sufficient awareness about elder abuse and its laws, which might be the main reason for their indifference toward the detection and reporting of this misconduct (20). In a study by Taylor and Dodd in Syria, the subjects showed low self-esteem in response to questions related to awareness about abuse, because they knew little about abuse and were very con- 


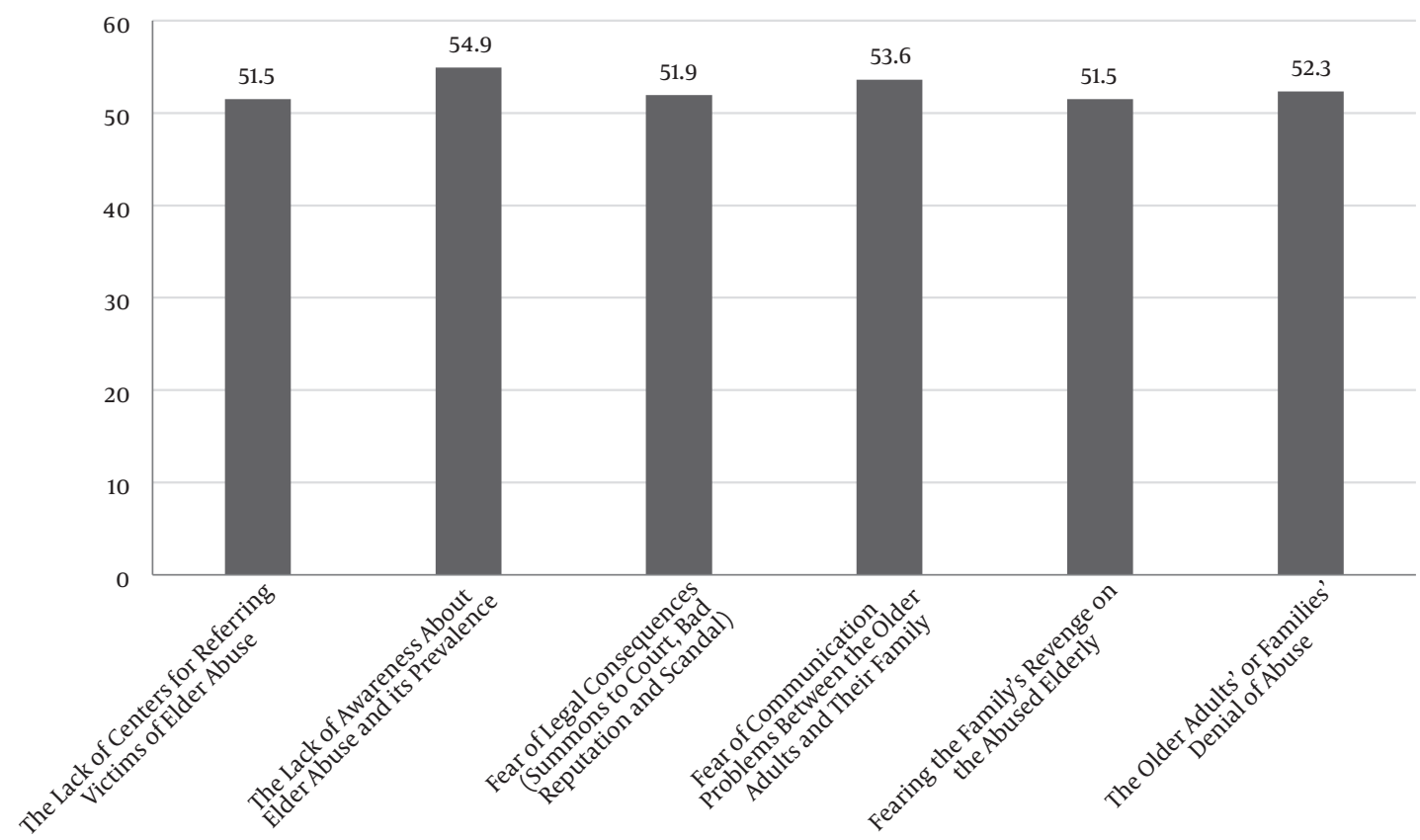

Figure 2. Challenges of healthcare providers' detection and report of elder abuse

\begin{tabular}{|c|c|c|c|}
\hline Items & Not Important at All & No Idea & Very Important \\
\hline Recent changes in rules and guidelines & $77(7.32)$ & $87(37)$ & $71(30.2)$ \\
\hline The lack of a professional protocol for assessing elder abuse & $56(8.23)$ & $80(34)$ & $99(42.1)$ \\
\hline The lack of a professional protocol for managing elder abuse & $61(9.25)$ & $71(2.30)$ & $103(43.8)$ \\
\hline The absence of centers for referring victims of elder abuse & $75(2.24)$ & $57(3.24)$ & $121(51.4)$ \\
\hline The lack of awareness about elder abuse and its prevalence & $48(4.20)$ & $58(7.24)$ & $129(54.8)$ \\
\hline Fear of legal consequences (summons to court, bad reputation and scandal) & $50(2.21)$ & $63(8.26)$ & $122(51.9)$ \\
\hline Fear of communication problems between the older adults and their family & $47(20.0)$ & $62(4.26)$ & $126(53.6)$ \\
\hline Fearing the family's revenge on the abused elderly & $53(5.22)$ & $61(26)$ & $121(51.48)$ \\
\hline The lack of time for evaluation, referral and follow-up & $62(3.26)$ & $74(31.5)$ & $99(42.1)$ \\
\hline Cultural/language barriers in communication with the older adults & $55(4.23)$ & $63(8.26)$ & $117(49.7)$ \\
\hline The older adults' or families' denial of abuse & $46(5.19)$ & $66(1.28)$ & $123(52.3)$ \\
\hline $\begin{array}{l}\text { The resistance of the older adults/families against abuse assessment, intervention and } \\
\text { management }\end{array}$ & $55(4.23)$ & $74(5.31)$ & $106(45.1)$ \\
\hline I have no personal interest in pursuing this issue & $57(2.24)$ & $100(6.42)$ & $78(33.1)$ \\
\hline The case not being a priority -Assessing elder abuse not being part of elder care programs & $58(6.24)$ & $78(2.33)$ & $99(42.1)$ \\
\hline
\end{tabular}

${ }^{\mathrm{a}}$ Values are expressed as No. (\%).

fused about what to construe as misconduct, given that there are no clear definitions or legal descriptions of misconduct. The study showed the positive impact of education on the awareness, beliefs, and attitudes of the personnel (21). Mclaoughlin et al., showed that comprehensive training programs are required to increase the awareness of social workers, health care providers, and other healthcare personnel about elder abuse as well as help identify different forms and methods of interventions to help eliminate this misconduct (22). Childs et al., declared that lack 


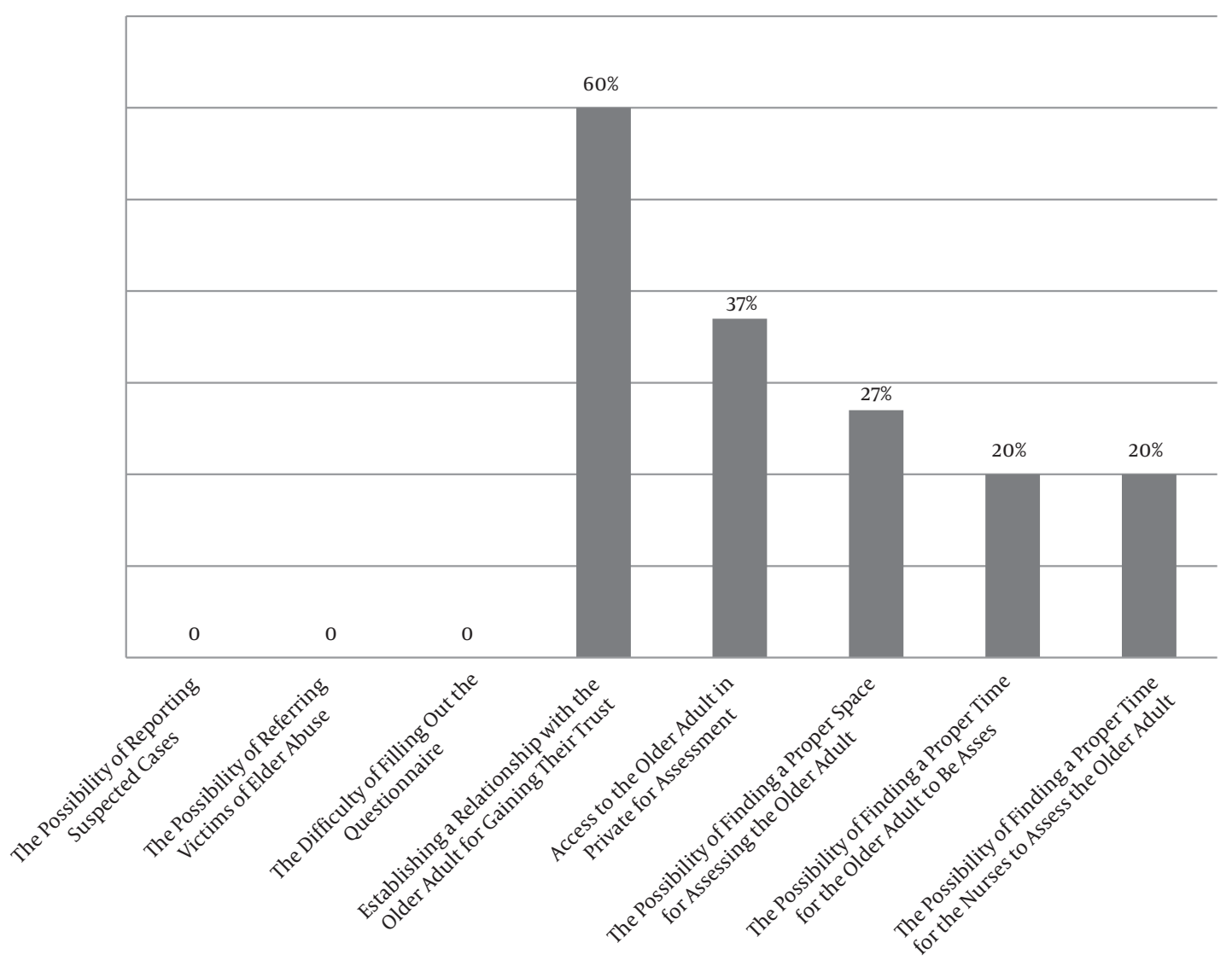

Figure 3. The of healthcare providers' implementation problems in elder abuse screening

of knowledge may prevent professionals from detecting abuse. Individual perceptions of abuse may also affect the judgment made about an offensive behavior (23). Kennedy showed that continuing education is required in the primary medical care sector to increase awareness about elder abuse and enable its early detection (24). Daly and Coffey reported that the lack of awareness can lead to misconduct in places that provide long-term care (25).

The findings revealed a significant relationship between the health care providers' awareness and age; that is, younger health care providers had a higher awareness. One of the reasons for this difference might be the greater use of technology and presence in the virtual world in the younger generation. Younger health care providers are also more eager to learn while older health care providers may not have enough time for further reading, due to their greater problems in life. Another reason for this difference is the greater curiosity of young health care providers and their ability to accept issues with reasoning rather than blindly. Younger health care providers may also face situations that raise questions for them because of being less experienced and may need to read more to get answers; thus, increasing their knowledge. The health care providers at Rouhani Hospital had a better attitude toward older adults. Health care providers with more work experience had a lower level of awareness.

Several studies have examined how demographic characteristics are related to the awareness about old age and the attitude toward older adults and have yielded somewhat different and scattered results, which could be due to their different methods of sampling, differences in the target groups, cultural differences in the study population, and different assessment tools used (26). Examining the barriers and causes of abuse in the present study revealed the lack of awareness about abuse and its prevalence, fear of legal consequences (summons to court, bad reputation, and scandal), and fear of communication problems between older adults and their family as the main barriers to the provision of elderly care, while the lack of personal interest in caring for older adults was the least ef- 
fective barrier. The findings obtained by Beaulaurier et al., in Florida showed that each internal attribute of the victim, such as inability, self-blame, secrecy, family protection, and frustration make the victim reluctant to seek help in cases of domestic elder abuse. These behaviors construe the internal barriers to detecting and helping cases of elder abuse (27). Chokkanathan et al., also stated that older adults believe their family reputation may be stained if they ask for help in cases of elder abuse; this reputation may affect the future marriage of their single daughters. In addition, they may be embarrassed by seeking help and may feel that being abused is their fate. Raising awareness through the mass media, posting publicity and messages about preventing elder abuse in places such as parks, which are visited by many people, and employing influential figures to communicate the message can be effective in identifying and eliminating these barriers. With the help of medical professionals, social workers, and other health care providers who are in contact with older adults, the elderly who consider being abused as their fate can receive help (28). Schmeidel et al., proposed some of the barriers to detecting and reporting elder abuse as job position, evaluation, interpretation, system, knowledge, and awareness. In this proposal, the emphasis on each of these categories varied among different professions. The findings suggested that each group of healthcare providers have different views about detecting and reporting elder abuse. Health care providers considered abuse unusual and said that they did not have enough time to assess their patients for detecting any misconduct.

Meanwhile, the physicians insisted on detecting and reporting cases of abuse and believed that maintaining their patients' trust helped in the detection of abuse. Social workers have a major role in detecting and reporting abuse due to their knowledge and experience in the field. One study showed that knowledge and awareness are crucial in the detection of elder abuse at all levels (29). The results of another study in the US showed that the lack of adequate knowledge and awareness about abuse and its signs are barriers to detecting and reporting instances of it, and that the awareness of physicians and other healthcare personnel should be raised through workshops and conferences (30). The barriers reported in other countries are consistent with the barriers reported in this study in Iran and any discrepancies are due to cultural differences and the different laws governing the countries. Training programs and workshops aiming to raise the awareness of health care providers and physicians can lead to a positive feedback and can be effective in preventing elder abuse.

The limitations of this study included the lack of cooperation with the researcher in some health care providers and the short duration of the workshops. Long-term and perhaps continuing training should be provided in future studies. Another limitation was that the study only emphasized empowering health care providers to identify abuse and did not include the management or care of the elder abuse. Future studies recommended to address the empowerment of health care providers from identification of elder abuse to care, and referral to support centers. The researchers also recommend studies on eliminating the barriers to health care providers' detection of elder abuse. Further research strategies should also be implemented to reduce elder abuse, such as national training programs to raise the public awareness about elder abuse, encouraging the cooperation of the IRIB to raise awareness in the society about old age and elder abuse, providing courses at all levels in schools and universities to help raise awareness about elder abuse (particularly in medical sciences universities), and extensive coordination and cooperation between the public and private sectors to develop programs that promote active aging. Further research should be focused on the planning to prevent and support the elder abuse.

\subsection{Conclusions}

The results showed that the awareness of the health care providers regarding the detection of elder abuse was low. The mean health care providers' awareness about detecting elder abuse improved after educational program. The challenges of health care providers for detection of elder abuse were; the lack of awareness about elder abuse and its prevalence, fear of legal consequences, and fear of communication problems between older adults and their family. The results suggest that designing a comprehensive interventional study is necessary for professional healthcare providers to timely identify elderly abuse and prevent its harmful outcomes.

\section{Acknowledgments}

The authors would like to express their gratitude to the Research Deputy of Babol University of Medical Sciences and the Social Determinants of Health Research Center for funding this project and also to the nurses at Rouhani and Shahid Beheshti hospitals for participating in the research. In addition, we would like to thank Dr. Evangeline Foronda for English editing.

\section{Footnotes}

Authors' Contribution: Study design: Shima Sum, Mahbobeh Faramarzi, and Akram Fallah Shojaei Mardomakdehi; data collection: Akram Fallah Shojaei Mar- 
domakdehi; manuscript writing: Shima Sum and Mahbobeh Faramarzi; All authors read and approved the final manuscript.

Conflict of Interests: There are no conflicts of interest. Ethical Approval: The project's compliance with the ethical code was approved by the Ethics Committee of Babol University of Medical Sciences (MUbabol.HRI.REC.1394.15).

Funding/Support: The Deputy of Research at Babol University of Medical Sciences approved and supported the study.

\section{References}

1. Heravi Karimooei M, Anousheh M. [The effect of family counseling programs to prevent abuse of elderly women]. Daneshvar. 2000;7(27):39-46. Persian.

2. Payne BK. An integrated understanding of elder abuse and neglect. $J$ Crim Justice. 2002;30(6):535-47. doi: 10.1016/s0047-2352(02)00175-7.

3. World Health Organization. Elder abuse. 2017. Available from: http:// www.who.int/ageing/projects/elder-abuse/en/.

4. Adib-Hajbaghery M, Aghahoseini S. The evaluation of disability and its related factors among the elderly population in Kashan, Iran. BMC Public Health. 2007;7:261. doi: 10.1186/1471-2458-7-261. [PubMed: 17888171]. [PubMed Central: PMC2039743].

5. Esmailzadeh S, Faramarzi M, Mosavi S. Prevalence and determinants of intimate partner violence in Babol city, Islamic Republic of Iran. East Mediterr Health J. 2005;11(5-6):870-9.

6. Faramarzi M, Esmailzadeh S, Mosavi S. A comparison of abused and non-abused women's definitions of domestic violence and attitudes to acceptance of male dominance. Eur J Obstet Gynecol Reprod Biol. 2005;122(2):225-31. doi: 10.1016/j.ejogrb.2004.11.047. [PubMed: 15935543].

7. Kissal A, Beser A. Elder abuse and neglect in a population offering care by a primary health care center in Izmir, Turkey. Soc Work Health Care. 2011;50(2):158-75. doi: 10.1080/00981389.2010.527570. [PubMed: 21347984].

8. World Health Organization. A global response to elder abuse and neglect: Building primary health care capacity to deal with the problem worldwide: Main report. Geneva, Printed in France: WHO Press; 2008.

9. Heravi-Karimooi M, Anoosheh M, Foroughan M, Sheykhi MT, Hajizadeh E. [Designing and Determining Psychometric Properties of the Domestic Elder Abuse Questionnaire]. Salmand: Iran J Age. 2010;5(1):7-21. Persian.

10. Kheirkhah F, Poorkarim K, Hosseini SR, Bijani A, Parsian H, Hamidia $A$, et al. The association between zinc and cognitive impairment in elderly people of Iran. Shiraz E-Med J. 2017;18(7). e13093. doi: 10.5812/semj.13093.

11. Faramarzi M, Cheraghi M, Zamani M, Kheirkhah F, Bijani A, Hosseini SR. Gender-specific predictors of depressive symptoms among community elderly. I Res Health Sci. 2017;17(2). e00377. [PubMed: 28469046]

12. Rastegarpur SA. [Planning and health service management of elderly in Tehran in 1376, collected articles on aging]. Tehran: Charity Women Publication; 2001. Persian.

13. Janmardi M. [Assessing the issues and problems of elderly in Saghez city with sociological approach. collected articles on aging (issues of society and environment)]. 2. Tehran: Charity Women Publication; 1999. Persian.
14. Manoochehri H, Ghorbi B, Hosseini M, Nasiri Oskuyee N, Karbakhsh $M$. Degree and types of domestic abuse in the elderly referring to parks of Tehran. SBMU Facul Nurs Midwifery Q. 2009;18:37-43. doi: 10.22037/anm.v18i63.1182.

15. Criner JA. The nurse's role in preventing abuse of elderly patients. Rehabil Nurs. 1994;19(5):277-80. 297. doi: 10.1002/j.20487940.1994.tbo0822.x. [PubMed: 7855392].

16. Winterstein TB. Nurses' experiences of the encounter with elder neglect. J Nurs Scholarsh. 2012;44(1):55-62. doi: 10.1111/j.15475069.2011.01438.x. [PubMed: 22340016].

17. Phelan A. Elder abuse and the community nurse: Support ing the patient. $\mathrm{Br} J$ Community Nurs. 2010;15(10):472-8. doi 10.12968/bjcn.2010.15.10.78727. [PubMed: 20966841].

18. Alon S, Berg-Warman A. Treatment and prevention of elder abuse and neglect: Where knowledge and practice meet-a model for intervention to prevent and treat elder abuse in Israel. J Elder Abuse Negl. 2014;26(2):150-71. doi: 10.1080/08946566.2013.784087. [PubMed: 24499281]

19. Fisher JM, Rudd MP, Walker RW, Stewart J. Training tomorrow's doctors to safeguard the patients of today: Using medical student simulation training to explore barriers to recognition of elder abuse. J Am Geriatr Soc. 2016;64(1):168-73. doi: 10.1111/jgs.13875. [PubMed: 26782868].

20. Almogue A, Weiss A, Marcus EL, Beloosesky Y. Attitudes and knowledge of medical and nursing staff toward elder abuse. Arch Gerontol Geriatr. 2010;51(1):86-91. doi: 10.1016/j.archger.2009.08.005. [PubMed: 19775762].

21. Taylor K, Dodd K. Knowledge and attitudes of staff towards adult protection. J Adult Protect. 2003;5(4):26-32. doi: $10.1108 / 14668203200300031$.

22. McLaughlin J, Lavery H. Awareness of elder abuse among community health and social care staff in Northern Ireland: An exploratory study. J Elder Abuse Neglect. 2000;11(3):53-72. doi:10.1300/J084v11n03_04.

23. Childs HW, Hayslip B Jr, Radika LM, Reinberg JA. Young and middleaged adults' perceptions of elder abuse. Gerontologist. 2000;40(1):7585. doi: 10.1093/geront/40.1.75. [PubMed: 10750315].

24. Kennedy RD. Elder abuse and neglect: The experience, knowledge, and attitudes of primary care physicians. Fam Med. 2005;37(7):481-5. [PubMed: 15988632].

25. Daly J, Coffey A. Staff perceptions of elder abuse. Nurs Older People. 2010;22(4):33-7. doi: 10.7748/nop2010.05.22.4.33.c7735. [PubMed: 20503678].

26. Soderhamn O, Lindencrona C, Gustavsson SM. Attitudes toward older people among nursing students and registered nurses in Sweden. Nurse Educ Today. 2001;21(3):225-9. doi: 10.1054/nedt.2000.0546. [PubMed: 11322814].

27. Beaulaurier RL, Seff LR, Newman FL, Dunlop BD. Internal barriers to help seeking for middle-aged and older women who experience intimate partner violence. J Elder Abuse Negl. 2005;17(3):53-74. doi: 10.1300/j084v17n03_04. [PubMed: 16931469].

28. Chokkanathan S, Natarajan A, Mohanty J. Elder abuse and barriers to help seeking in Chennai, India: A qualitative study. J Elder Abuse Negl. 2014;26(1):60-79. doi: 10.1080/08946566.2013.782786. [PubMed: 24313798].

29. Schmeidel AN, Daly JM, Rosenbaum ME, Schmuch GA, Jogerst GJ Health care professionals' perspectives on barriers to elder abuse detection and reporting in primary care settings. J Elder Abuse Negl. 2012;24(1):17-36. doi: 10.1080/08946566.2011.608044. [PubMed 22206510]. [PubMed Central: PMC3298114].

30. Taylor DK, Bachuwa G, Evans J, Jackson-Johnson V. Assessing barriers to the identification of elder abuse and neglect: A communitywide survey of primary care physicians. J Natl Med Assoc. 2006;98(3):403-4. [PubMed: 16573305]. [PubMed Central: PMC2576103]. 